\title{
34. Prophylactic and Therapeutic Effect of Bactobolin on Autoimmune Encephalomyelitis
}

\author{
By Takeshi TabIRA,*) Yao Da-Lin,*) Takashi Yamamura,*) \\ and Takaaki AoYAGI**)
}

(Communicated by Setsuro Ebashi, M. J. A., April 13, 1987)

Summary. Bactobolin, a novel antineoplastic and antibiotic, was tested for prevention and treatment of experimental autoimmune encephalomyelitis (EAE) in Lewis rats. Clinical and histological signs of EAE induced by active challenge with myelin basic protein and Freund's complete adjuvant were profoundly suppressed by prophylactic treatment with bactobolin during the inductive phase of EAE in association with diminished lymphocyte proliferative response to sensitized antigens. It was also effective on the effector phase of actively induced EAE as well as passively induced EAE with primary cultured spleen cells sensitized to myelin basic protein. Moreover, therapeutic administration of bactobolin after onset of EAE was effective. The results suggest possible usefulness of bactobolin for treatment of autoimmune disease.

Introduction. In spite of refinement in understanding the mechanism of autoimmune diseases, most of them are still incurable and effort of screening new drugs has been continued using animal models. Experimental autoimmune encephalomyelitis (EAE) has greatly contributed to the study of neuroimmunological processes probably involved in the human demyelinating diseases such as postinfectious encephalomyelitis and multiple sclerosis. ${ }^{1)}$ It has also served as an excellent model for studying the treatment of $\mathrm{T}$ cell-dependent autoimmune disease. If this experimental system is adopted for the selection of immune modulating compounds to be used in the therapy of established autoimmune disease in human, agents capable of effecting a cure rather than those only preventing the induction of the disease are required. Recently several new agents with therapeutic effect after onset or with preventing effect on the effector phase of EAE have been reported; these are protease inhibitors, ${ }^{2)}$ serum thymic factor, ${ }^{3)}$ monoclonal anti-L3T4 or anti-Ia antibody, ${ }^{4), 5)}$ cyclosporin $\mathrm{A},{ }^{6)}$ desferrioxamine,,$^{7}$ mitoxantrone, ${ }^{8)}$ prazosin, ${ }^{9)}$ 15-deoxyspergualin ${ }^{10)}$ and others. In our effort of screening new drugs, we found bactobolin has superior effect on not only prevention but also treatment of EAE.

Materials and methods. Female Lewis rats, weighing approximately $200 \mathrm{~g}$, were used throughout the experiment. The antigen, myelin basic protein (BP), was prepared from guinea pig brain and spinal cord.

For active induction of EAE, rats were injected into footpads with $0.1 \mathrm{ml}$ of an emulsion containing $50 \mu \mathrm{g} \mathrm{BP}$ and $200 \mu \mathrm{g}$ Mycobacterium tuberculosis, H37Ra, in complete Freund's adjuvant (CFA). To induce passive form of EAE,

*) Division of Demyelinating Disease and Aging, National Institute of Neuroscience, National Center of Neurology and Psychiatry, 4-1-1 Ogawa-Higashi, Kodaira, Tokyo 187, Japan.

**) Institute of Microbial Chemistry, 3-14-23 Kamiosaki, Shinagawa, Tokyo 141, Japan. 
[Vol. 63(B),

animals were immunized similarly, and on day 11 spleen cells were prepared, cultured in the presence of $10 \mu \mathrm{g} / \mathrm{ml} \mathrm{BP}$ for 3 days, and $2 \times 10^{\tau}$ of the primary cultured cells were inoculated into the naive Lewis rats via tail vein.

For lymphocyte proliferation assay, the draining lymph nodes of selected animals were removed 11 days after immunization (day 11). The lymph node cells were suspended at $4 \times 10^{6} / \mathrm{ml}$ in conditioned medium including $5 \%$ fetal calf serum, and cultured with BP $(10 \mu \mathrm{g} / \mathrm{ml})$, purified protein derivative (PPD) $(10 \mu \mathrm{g} / \mathrm{ml})$, or concanavalin A $(2 \mu \mathrm{g} / \mathrm{ml})$ for $72 \mathrm{hrs}$. These cells were labelled with ${ }^{3} \mathrm{H}$-methyl-thymidine $(1 \mu \mathrm{Ci} /$ well $)$ for the final $18 \mathrm{hrs}$.

The clinical sign of EAE was daily graded from 0 to 5 : 0 , healthy; 1 , tail atony; 2, mild paraparesis; 3 , paraplegia; 4, quadriparesis; 5 , moribund. The severity of EAE in each case was also expressed as "paralysis score", the summation of daily clinical grade during illness. Histopathologic study was performed with formalin-fixed and paraffin-embedded tissues and the lesions were graded from 0 to $4: 0$, no lesion; 1 , lymphocyte cuffing limited to perivascular space; $2-4$, mild, moderate and severe infiltration of inflammatory cells into the central nervous system parenchyma.

Bactobolin was dissolved in sterile phosphate buffered saline (PBS) at $0.2 \mathrm{mg} / \mathrm{ml}$, and injected daily into the peritoneal cavity of the rats. Control animals were injected with $0.5 \mathrm{ml}$ of PBS.

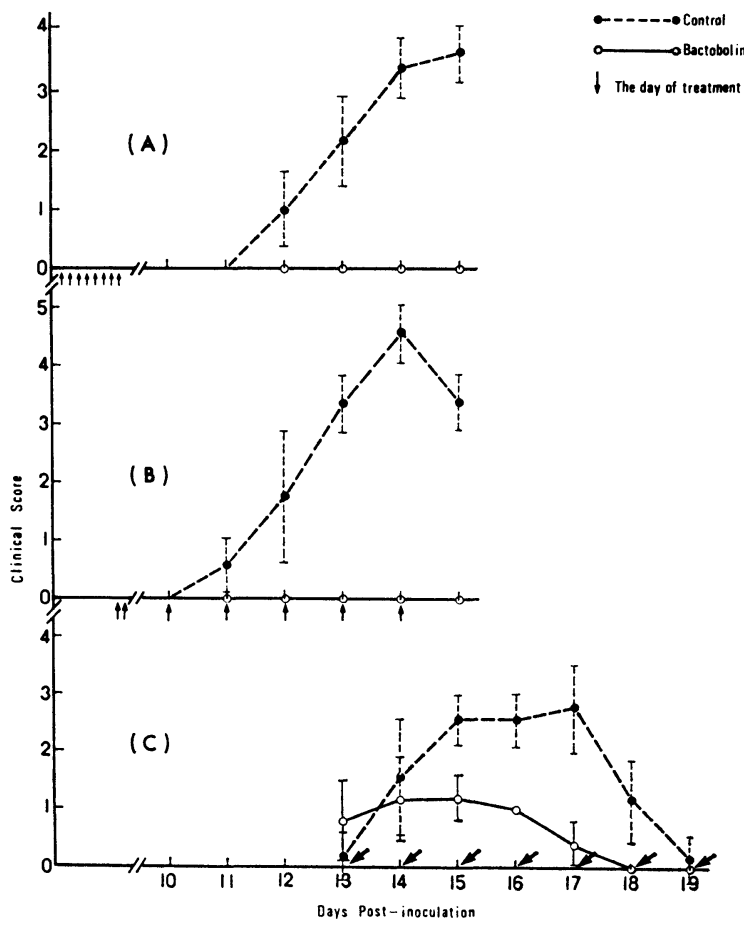

Fig. 1. Prevention and therapy of EAE with bactobolin. A: Treatment during the induction phase of EAE (day 1 to 8), when effector T cells are generated. B: Treatment during the effector phase of EAE (day 8 to 14 ), when already generated effector $T$ cells maturate. C: Treatment after onset of clinical EAE. As shown, bactobolin is very effective on either stage. 
Results. Prevention of the inductive phase of EAE. Rats immunized with $\mathrm{BP}$ were treated with $0.5 \mathrm{mg} / \mathrm{kg} /$ day of bactobolin from day 1 to day 8 . All control animals treated with PBS alone developed severe EAE characterized by

Table I. Effect of bactobolin on the histologic Score*

\begin{tabular}{|c|c|c|}
\hline & $\mathrm{n}$ & Histologic score $(\bar{x} \pm \mathrm{SD})$ \\
\hline PBS & 6 & $3.0 \pm 0.3$ \\
\hline Bactobolin & 5 & $0.5 \pm 0.6$ \\
\hline \multicolumn{3}{|c|}{ B. Treatment during the effector phase $* *$} \\
\hline & $\mathrm{n}$ & Histologic score $(\overline{\mathrm{x}} \pm \mathrm{SD})$ \\
\hline PBS & 5 & $2.4 \pm 0.5$ \\
\hline Bactobolin & 5 & $0.1 \pm 0.1$ \\
\hline
\end{tabular}

* Histologic score is the mean of those for six separate sections, including spinal cord and brain. All animals were sacrificed on day 15 . ** Definition of induction phase and effector phase: see the legend to Fig. 1.

Table II. Effect of bactobolin on lymphocyte proliferative responses to antigens and mitogen*

\begin{tabular}{|c|c|c|}
\hline $\begin{array}{l}\text { Antigen or } \\
\text { mitogen }\end{array}$ & $\begin{array}{l}\text { Control group } \\
\mathrm{CPM} \pm \mathrm{SD}\end{array}$ & $\begin{array}{l}\text { Bactobolin group*** } \\
\text { CPM } \pm \mathrm{SD}\end{array}$ \\
\hline$(-)$ & $11,927 \pm 531$ & $5,858 \pm 316$ \\
\hline BP & $30,214 \pm 2,081$ & $2,507 \pm 670$ \\
\hline PPD & $54,554 \pm 2,958$ & $5,315 \pm 696$ \\
\hline Con A & $338,783 \pm 6,433$ & $32,271 \pm 27,295$ \\
\hline
\end{tabular}

* Lymph node cells were obtained from animals 11 days after immunization with $\mathrm{BP} / \mathrm{CFA}$, cultured with $\mathrm{BP}(10 \mu \mathrm{g} / \mathrm{ml})$, PPD $(10 \mu \mathrm{g} / \mathrm{ml})$ or con A $(2 \mu \mathrm{g} / \mathrm{ml})$ for 3 days. Proliferative responses were assessed by measuring the uptake of tritiated thymidine. ** Animals belonging to bactobolin group were daily injected with $0.5 \mathrm{mg} / \mathrm{kg}$ bactobolin from day 1 to day 8, and control animals were treated with PBS.

ascending paralysis from tail to the lower and upper limbs, incontinence and body weight loss. Histological examination on day 15 revealed severe inflammatory cell infiltration in the central nervous system. In contrast, none of the bactobolin-treated animals showed any clinical sign up to the day of sacrifice (day 15) (Fig. 1A). Histological lesion was also profoundly suppressed (Table I-A). Lymph node cells from these animals showed markedly suppressed proliferative response to $\mathrm{BP}, \mathrm{PPD}$ and concanavalin A (Table II).

Treatment of the effector phase of $E A E$. Animals were treated with bactobolin starting from day 8 , when effector $T$ cells capable of inducing EAE had already been generated. The result shows also marked suppression of EAE both clinically and histologically (Fig. 1B, Table I-B). To further refine this, we then treated passive EAE induced by the transfer of BP-sensitized and primary cultured spleen cells. As shown in Table III, the development of passive EAE was markedly suppressed by the treatment. 
Table III. Effect of bactobolin on passively induced EAE*

\begin{tabular}{lcc}
\hline $\begin{array}{c}\text { Treatment of } \\
\text { recipients }\end{array}$ & $\begin{array}{c}\text { Incidence of } \\
\text { clinical EAE }\end{array}$ & $\begin{array}{c}\text { Paralysis score } \\
(\overline{\mathrm{x}} \pm \mathrm{SD})\end{array}$ \\
\hline PBS & $2 / 2$ & $9.5 \pm 0.5$ \\
Bactobolin $(0.5 \mathrm{mg} / \mathrm{kg})$ & $0 / 2$ & $0 \pm 0$ \\
\hline
\end{tabular}

* Each recipient was transferred with $2 \times 10^{7}$ precultured spleen cells obtained from donor rats 11 days after immunization with BP/CFA. ** The recipients were treated from day 1 to day 8 after transfer and sacrificed on day 9 . *** The paralysis score is the cumulative clinical score.

Treatment after onset of EAE. Finally we tested the therapeutic effect of bactobolin starting on the day of onset of the disease. It did not shorten the duration of the disease but significantly suppressed the severity (Fig. 1C).

In all experiments, none of the bactobolin-treated animals showed significant pathologic change in the general organs except for atrophy of the thymus.

Discussion. Bactobolin is a product of Pseudomonas BMG 13-A7, and it inhibited the growth of Gram-positive and Gram-negative bacteria and experimental animal tumors. ${ }^{11}$ ) The inhibition was thought to be due to inhibition of protein synthesis. ${ }^{12)}$ We know its suppressing effect on antibody production, ${ }^{13)}$ but the suppression mechanism of EAE is not known yet.

The most striking feature of bactobolin is its favorable effect on not only prevention but also treatment of the disease after onset. We have compared the effect with dexamethasone and found bactobolin has much stronger therapeutic effect (data not shown). Among the new agents listed before, cyclosporin A has similar effect but it did not suppress passively induced EAE. ${ }^{14)}$ Because of its superior effect on autoimmune encephalomyelitis, further investigation is warranted for the possible use in human autoimmune diseases.

Acknowledgements. The authors express thanks to Dr. Tomio Takeuchi, Director of the Institute of Microbial Chemistry for valuable discussions. This study was partly supported by a grant-in-aid for New Drug Development Research, and by a grant from the Neuroimmunological Research Committee, the Ministry of Health and Welfare, Japan.

\section{References}

1) Alvord, E. C. Jr. et al.: Experimental Allergic Encephalomyelitis-A Useful Model for Multiple Sclerosis. Alan R. Liss, New York (1984).

2) Brosnan, C. F. et al.: Nature, 285, 235-237 (1980).

3) Nagai, Y. et al.: Japan. J. Exp. Med., 52, 213-219 (1982).

4) Waldor, M. K. et al.: Science, 227, 415-417 (1985).

5) Sriram, S., and Steinman, L.: J. Exp. Med., 158, 1362-1367 (1983).

6) Bolton, C. et al.: J. Neurol. Sci., 56, 147-153 (1982).

7) Bowern, N. et al.: J. Exp. Med., 160, 1532-1543 (1984).

8) Levine, S., and Saltzman, A.: J. Neuroimmunol., 13, 175-181 (1986).

9) Brosnan, C. F. et al.: J. Immunol., 137, 3451-3456 (1986).

10) Yamamura, T. et al.: J. Neurol. Sci. (1987) (submitted).

11) Kondo, S. et al.: J. Antibiotics, 32, 1069-1071 (1979).

12) Hori, M. et al.: ibid., 34, 465-468 (1981).

13) Ishizuka, M. et al.: ibid., 33, 1054-1062 (1980).

14) Hinrichs, D. J. et al.: Cell. Immunol., 77, 202-209 (1983). 